\title{
Structural and mechanical properties of AZOY thin films deposited on flexible substrates
}

\author{
C. Oliveira ${ }^{1, a}$, C. M. Costa ${ }^{1, b}$, L. Rebouta ${ }^{1, c}$, T. Viseu ${ }^{1, d}$, T. de Lacerda- \\ Arôso $^{1, \mathrm{e}}$, S. Lanceros-Mendez ${ }^{1, \mathrm{f}}$, E. Alves ${ }^{2, \mathrm{~g}}$ \\ ${ }^{1}$ Dep. Física, Universidade do Minho, 4710-057 Braga, Portugal \\ ${ }^{2}$ ITN, Dep. Física, EN. 10, 2686-953 Sacavém, Portugal \\ acadoliveira@gmail.com; ${ }^{b} \mathrm{cmscosta@fisica.uminho.pt,}{ }^{\mathrm{C}}$ rebouta@fisica.uminho.pt; \\ dtviseu@fisica.uminho.pt; ${ }^{\mathrm{d}}$ tla@fisica.uminho.pt; 'lanceros@fisica.uminho.pt; ${ }^{9}$ ealves@itn.mces.pt
}

Keywords: Al doped $\mathrm{ZnO}$, transparent conducting oxide, magnetron sputtering, electroactive polymers

\begin{abstract}
.
Transparent conducting $\mathrm{Al}$ doped $\mathrm{ZnO}$ films have been deposited by dc magnetron sputtering on glass and polymer substrates at room temperature. Depositions have been carried out from an AZOY (contains a small amount of $\mathrm{Y}_{2} \mathrm{O}_{3}$ in addition to $\mathrm{Al}_{2} \mathrm{O}_{3}$ and $\mathrm{ZnO}$ ) target under different conditions such as working pressure, substrate bias voltage and oxygen flow rate. The crystallinity of the $\mathrm{Al}$ doped $\mathrm{ZnO}$ films has been improved by using low-energy-ion bombardment. Likewise, the use of either the rotation or the static mode of the substrate during deposition influences the crystallinity and therefore the optical parameters and the electrical resistance of the films. Increasing the thickness of the films reduces the threshold strain at which the films can be deformed without provoking significant changes on their electrical properties.
\end{abstract}

\section{Introduction}

Thin films of transparent conductive oxides (TCO) deposited by several techniques have a broad range of applications in optoelectronics, piezoelectric transducers and gas sensors among others. For this reason, TCO coatings have lately been the target of exhaustive studies [1-4]. The most commonly studied TCO material is ITO (indium tin oxide). However, the disadvantages concerning indium -the fact of being rare, toxic and expensive- have raised new alternative materials like zinc oxide. When doped with Al, Ga or In this oxide improves its electrical properties [3-6].

The main purpose of this work is the deposition of transparent conducting oxide films on polymers with electro-mechanical and electro-optical properties, in order to develop applications such as flexible touch screens and keyboards. The main role of TCO films in such applications is the reading of the electrical signals generated by the electroactive polymer, although keeping the transparency of the piezoactive ensemble. The electroactive polymer used as substrate for this investigation is poly-(vinilidene fluoride) - PVDF, with a thickness of $28 \mu \mathrm{m}$. This polymer does not allow temperatures higher than $70^{\circ} \mathrm{C}$, which brings new challenges for the deposition of the TCO films. In order to optimize the TCO films to be deposited on the electroactive polymer substrate, their transmittance in the visible range and their electrical conductivity has been investigated. The formation and density of cracking failures as well as the evolution of the sheet resistance and optical transmittance have been measured on film-polymer systems subjected to different mechanical solicitations.

\section{Experimental Details}

The films have been deposited under a mixed $\mathrm{Ar} / \mathrm{O}_{2}$ atmosphere, by dc reactive magnetron sputtering, from a ZnO:Al (AZOY) target, simultaneously on glass and on poly-vinilidene fluoride (PVDF) substrates. In order to check the influence of ion bombardment, and thus the energy 
delivered to the growing films, the bias voltage has been varied from 0 to $-30 \mathrm{~V}$. Extra circular solenoid coils have been used, and placed between the target and the substrate holder. The external magnetic field created by the dc current passing through the coils allowed the variation of the ion flux towards the substrate. A target current of $0.2 \mathrm{~A}$ has been used in all depositions, which corresponds to a current density of $2.5 \mathrm{~mA} \mathrm{~cm}^{-2}$. This current density has been adjusted in order to avoid substrate heating. Further, this current density is enough to limit the influence of oxygen partial base pressure in the conductivity of the films. The target-to-substrate distance has been kept constant at $8 \mathrm{~cm}$ in all runs and the substrate has not been heated during deposition. Deposition conditions are given in table 1.

Table 1 - Deposition parameters

\begin{tabular}{|l|l|}
\hline Deposition Parameter & \multicolumn{1}{|c|}{ Values } \\
\hline Argon flux [sccm] & $30-50-70$ \\
\hline Time [s] & $60-120-180$ \\
\hline Rotation [rpm] & $0-5$ \\
\hline Bias voltage [V] & $0-30$ \\
\hline Base pressure [Pa] & $2 \times 10^{-4}$ \\
\hline Working pressure [Pa] & $3-5 \times 10^{-1}$ \\
\hline
\end{tabular}

X-ray diffraction (XRD) has been used to examine the crystallinity and crystal orientation using $\mathrm{Cu} \mathrm{K} \alpha$ radiation (Philips PW 1710 apparatus). Rutherford Backscattering Spectrometry (RBS) has been carried out to access the atomic composition of the samples using the RUMP code [7]. RBS has been performed using a $\mathrm{He}^{+}$beam with $2 \mathrm{MeV}$ of energy. Spectral transmittance of the films has been measured by UV-Vis-NIR Spectrophotometer (Shimadzu UV 3101 PC) in the range 200 $\mathrm{nm}-1000 \mathrm{~nm}$. The electromechanical tests have been carried out on $1 \times 10 \mathrm{~cm}^{2}$ samples with a computer-controlled tensile testing machine (Minimat, Polymer Labs), the displacement speed being $0.5 \mathrm{~mm} / \mathrm{min}$. During these tests, electrical resistance has been measured as a function of deformation with an Agilent 34401A digital multimeter. The surface failure modes of the samples have been analyzed after the electromechanical tests, by Scanning Electron Microscopy (SEM) in a Leica S360 system.

\section{Results and Discussion}

The analysis of RBS spectra revealed an Al content of about 2 at.\%. The thickness of the films has been calculated from the real atomic density (at $\left./ \mathrm{cm}^{2}\right)$, making use of the theoretical density (5,6 $\mathrm{g} / \mathrm{cm}^{3}$ ). From these calculations, the deposition rate in static mode is found to be about $33 \mathrm{~nm} / \mathrm{min}$ while in rotation mode it is about $12 \mathrm{~nm} / \mathrm{min}$. In Fig. 1 are displayed the RBS spectra for two samples with thicknesses of approximately $68 \mathrm{~nm}$ (static) and $115 \mathrm{~nm}$ (rotation).

The AZOY films prepared by static mode revealed poor crystallinity [8] with a preferred $<002>$ orientation while the sample prepared by rotation mode has higher crystallinity, as shown by the Xray diffraction pattern displayed in Fig. 2. The only difference between these films is that while one had been deposited under substrate rotation, for the other, the substrate had remained static. In fact, in rotation mode the deposition rate is about $1 / 3$ of that in static mode, allowing a stronger influence of the low energy ion bombardment. The increase of the low-energy-ion bombardment during film growth is an effective way to change the nucleation and growth kinetics [9], promoting the surface mobility, what justifies the improved crystallinity.

Also, the energy delivered to the growing film per condensing particle has influence in the average crystallite size. From Fourier analysis of X-ray diffraction peaks [10], calculation of the grain size has been performed. The films prepared in static mode have an average crystallite size 
around $11 \mathrm{~nm}$, while those prepared by rotation mode have an average crystallite size around 15 $\mathrm{nm}$.

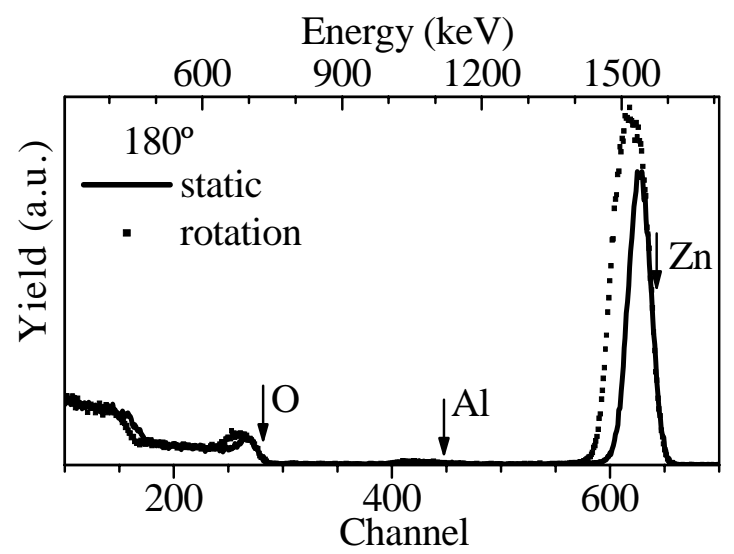

Fig. 1 - RBS spectra from two samples, one obtained in static and the other in rotation mode. The deposition parameters were the same.

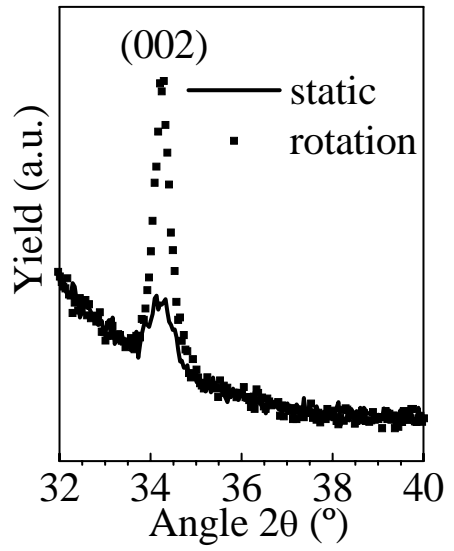

Fig. 2 - XRD spectra of the samples shown in Fig. 1.

Transmittance spectra for different samples are shown in Fig. 3. Each figure shows typical results where only one deposition parameter has been changed. As shown, the transparency on the visible range is affected by the deposition parameters. A thickness variation from $33 \mathrm{~nm}(\mathrm{t}=60 \mathrm{~s})$ to $68 \mathrm{~nm}(\mathrm{t}=120 \mathrm{~s})$ influences the spectral transmittance: thicker films became less transparent, as can be seen in Fig. 3-b). The application of a low bias voltage (-30V) to the substrate is useful (Fig. 3-c) as it increases the values of transmittance, though higher bias voltage results in films with inconvenient compressive stress. As well as in the bias voltage, altering the working pressure (Fig. 3 a) has changed spectral transmittance, but not significantly the electrical resistance. The motion mode of the substrate is also an important parameter since it has a strong influence in transmittance (Fig. $3 \mathrm{~d}$ ) as well as in conductivity.
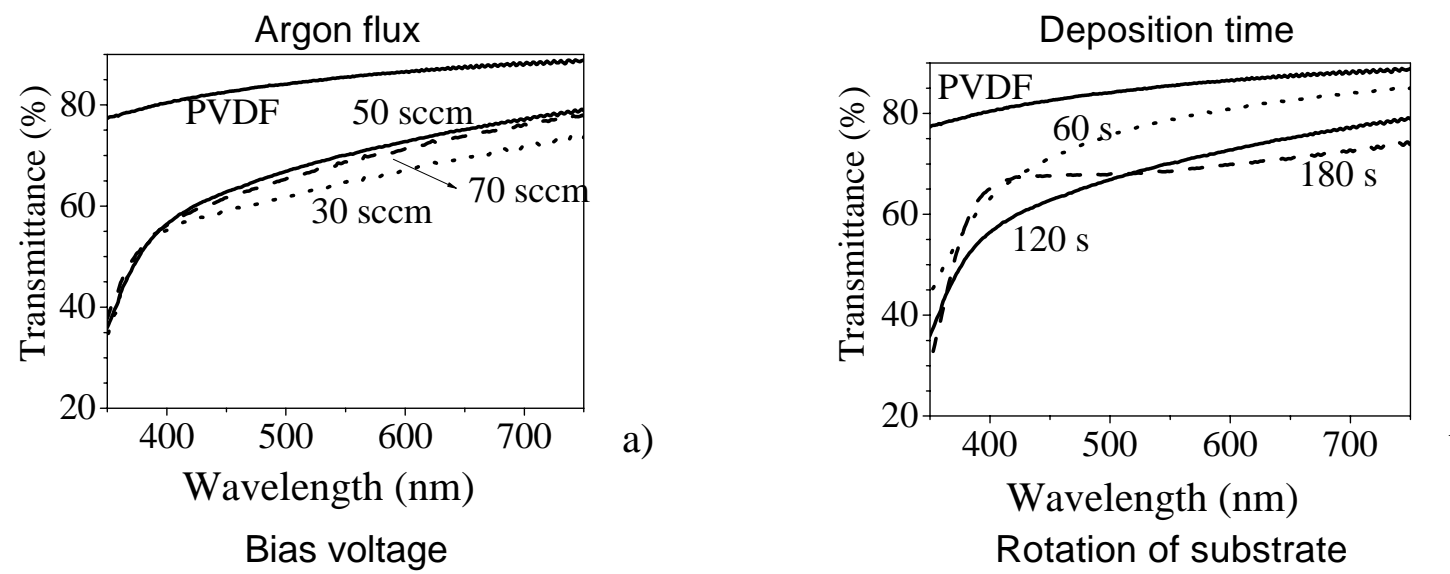

b)
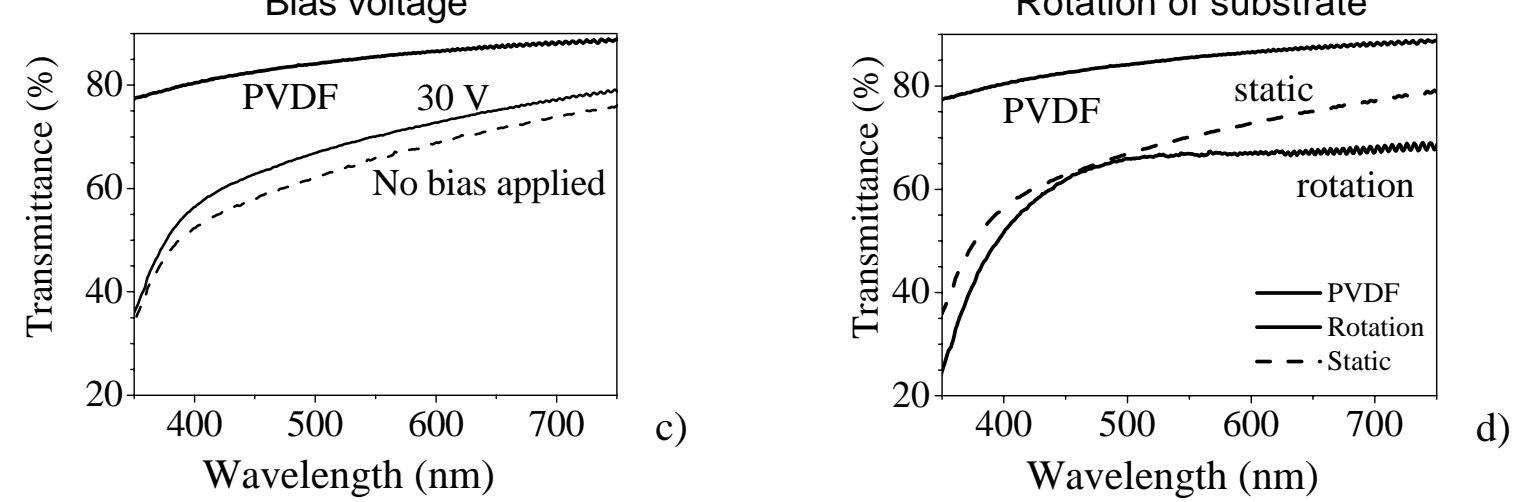

Fig. 3 - Spectral transmittance of substrate (PVDF) and samples prepared under different deposition conditions. In each figure all deposition parameters have been kept constant, except those indicated. 
All transmittance spectra have been analysed: thickness and optical parameters of the films have been determined by fitting the spectra using the Minkov[11] method and adopting a mono oscillator behaviour for the dispersion of the dielectric constant. All films have effective refractive index of about 1.95 at $550 \mathrm{~nm}$ (Fig. 4) that slightly decrease with decreasing thickness.

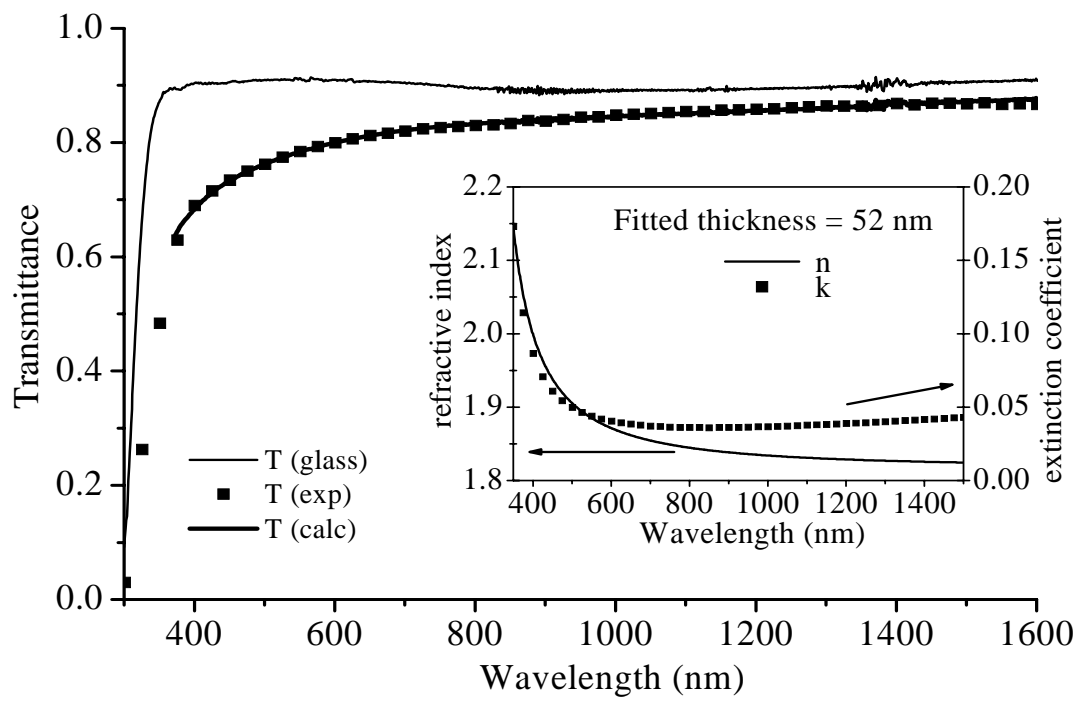

Fig. 4 - Transmission spectra (experimental data and fit) of a typical AZOY thin film deposited on glass. Glass transmittance is also shown. The refractive index and the extinction coefficient of the AZOY film obtained from the fit are shown in the inset.

Tensile stress applied to different samples revealed distinct failure modes, as shown in Fig. 5.
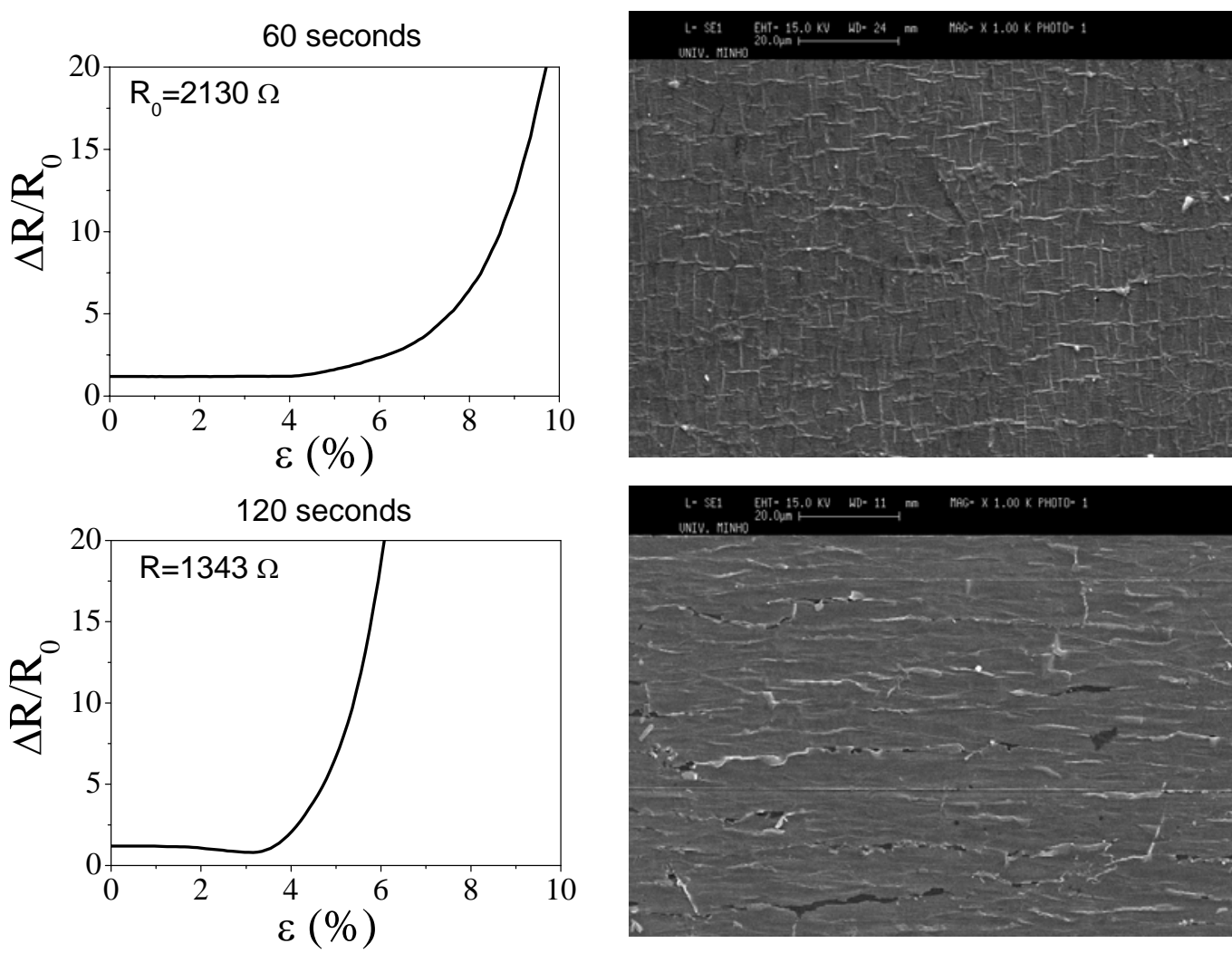

Fig. 5 - Evolution of the resistance as a function of the deformation and SEM images taken after the electromechanical tests at a deformation of $10 \%$. 
Here are displayed the top view SEM micrographs obtained after the test and the plots of the electrical resistance as a function of the deformation of the films deposited on PVDF substrates. The initiation of the tensile failure is affected by the physical properties of the samples. The curvature radius of the substrate was not changed, which means that the residual stress of the system is negligible when compared to its cohesive strength.

The increase in the resistance is due to the formation of cracks created by the applied load and it is related with the cohesive strength of the film. In samples shown in Fig. 5, it was found a direct correlation between the thickness of the deposited films (directly related to the deposition time) and the minimum deformation at which the crack propagation is associated with a substantial resistance increase.

\section{Summary}

Deposition parameters have influence on the physical properties such as optical transmittance and electrical resistance of AZOY thin films deposited in polymer substrates. The low substrate temperature during deposition has resulted in films with poor crystallinity, which decreases the conductivity.

For the same thickness and using the same deposition parameters, the samples prepared in rotation mode have lower resistance, slightly lower spectral transmittance as well as higher crystallinity than those prepared in static mode.

A direct correlation between the thickness of the films and the deformation at which the propagation of the cracks induce a substantial resistance increase has been found.

\section{Acknowledgements}

The authors thank the Portuguese Foundation for Science and Technology - FCT: POCI/CTM/59425/2004 and PTDC/CTM/69362/2006, co-financed by European community fund FEDER.

\section{References}

[1] I. Hamberg, C.G. Granqvist: J. Appl. Phys. Vol. 60 (1986), p.R123

[2] J.L. Huang, Y.T Jah, B.S. Yau, Ch.Y. Chen, H.H. Lu: Thin Solid Films Vol.370 (2000), p. 33

[3] V. Assunção, E. Fortunato, A. Marques, H. Aguas, I. Ferreira, M.E.V. Costa, R. Martins: Thin Solid Films Vol. 427 (2003), p. 401

[4] D. G. Lim, D.H. Kim, J. K. Kim, O. Kwon, K. J. Yang, K. I. Park, B. S. Kim, S. W. Lee, M. W. Park, D. J. Kwak; Superlattices and Microstructures Vol. 39 (2006), p. 107

[5] M. Fonrodona, J. Escarré, F. Villar, D. Soler, J.M. Asensi, J. Bertomeu, J. Andreu: Solar Energy Mater. \& Solar Cells Vol 89 (2205) p. 37

[6] K.H. Kim, R.A. Wibowo and B. Munir: Mater. Lett. Polymer Vol. 21 (2006), p. 1391

[7] L. R. Doolittle: Nucl. Inst. and Meth., B Vol. 9 (1985), p. 344.

[8] Su-Shia Lin, Jow-Lay Huang, P. Sajgalik: Surf. Coat. Technol. Vol. 190 (2005), p. 39

[9] J.E. Greene, S.A. Barnett, J.E. Sundgren and A. Rockett, Ion beam assisted film growth, Elsevier, Amesterdam, Elsevier, 1988.

[10] S. Krumm: Mater. Sci. Forum Vols. 228-231 (1996), p. 183

[11] D. A. Minkov, J. Phys D: Appl. Phys. 22 (1989), p 199 